PROCEEDINGS OF THE

AMERICAN MATHEMATICAL SOCIETY

Volume 130, Number 4, Pages 1125-1130

S 0002-9939(01)06194-9

Article electronically published on October 1, 2001

\title{
SEQUENTIALLY INDEPENDENT EFFECTS
}

\author{
STAN GUDDER AND GABRIEL NAGY
}

(Communicated by Joseph A. Ball)

\begin{abstract}
A quantum effect is a yes-no measurement that may be unsharp. An effect is represented by an operator $E$ on a Hilbert space that satisfies $0 \leq E \leq I$. We define effects $E_{1}, E_{2}, \ldots, E_{n}$ to be sequentially independent if the result of any sequential measurement of $E_{1}, E_{2}, \ldots, E_{n}$ does not depend on the order in which they are measured. We show that two effects are sequentially independent if and only if they are compatible. That is, their corresponding operators commute. We also show that three effects are sequentially independent if and only if all permutations of the product of their corresponding operators coincide. It is noted that this last condition does not imply that the three effects are mutually compatible.
\end{abstract}

\section{INTRODUCTION}

Sequential measurements are very important in quantum mechanics. In general, two measurements $M_{1}, M_{2}$ cannot be performed simultaneously so they are usually executed sequentially. Thus, they are either executed in the order $\left(M_{1}, M_{2}\right)$ or $\left(M_{2}, M_{1}\right)$. We may think of $\left(M_{1}, M_{2}\right)$ as a sequential measurement in which $M_{1}$ is measured first and $M_{2}$ second. As is frequently done, both experimentally and theoretically, we shall simplify the situation by considering yes-no measurements. These are measurements that only have two possible results, usually taken to be 0 and 1. A paradigm situation is an optical bench in which a beam of particles prepared in a certain state is injected at the left and then subjected to a sequence of filters $F_{1}, \ldots, F_{n}$. Particles that pass through all the filters enter a detection device at the right of $F_{n}$. In general, the order of placement of the filters makes a difference. For example, let $F_{1}, F_{2}, F_{3}$ be polarizing filters in planes perpendicular to the particle beam, where $F_{1}$ polarizes vertically, $F_{2}$ horizontally and $F_{3}$ at a $45^{\circ}$ angle. If we place the filters in order $F_{1}, F_{2}, F_{3}$, then no particles are detected, but in the order $F_{1}, F_{3}, F_{2}$ particles are detected. The difference is due to quantum interference and this phenomenon occurs for many other quantum measurements such as spin measurements in which the filters are given by Stern-Gerlach apparata.

A yes-no measurement may be thought of as an event that occurs when the measurement has value 1 and does not occur when the measurement has value 0 . In classical probability theory, events are represented by sets and the sequential

Received by the editors September 19, 2000 and, in revised form, October 27, 2000.

2000 Mathematics Subject Classification. Primary 47B15, 47B65; Secondary 81P10, 81P15.

Key words and phrases. Sequential independence, measurements, effects, positive operators, quantum mechanics.

The second author was partially supported by NSF grant DMS 9706858 .

(C)2001 American Mathematical Society 
measurement $(A, B)$ occurs with probability $P(A, B)=P(A) P(B \mid A)$. But $P(B \mid A)=P(A \cap B) / P(A)$ so that $P(A, B)=P(A \cap B)$. (We are assuming that $P(A) \neq 0$ which we shall always do in the sequel.) In this case, $P(A, B)=P(B, A)$ so there is no interference between $A$ and $B$ and this is one of the main differences between classical and quantum probability theory. Another way of stating this is that the sequential measurement $(A, B)$ is represented by the event $A \cap B$ and $(A, B)=(B, A)$ because $A \cap B=B \cap A$.

In quantum theory, sharp quantum events are represented by (self-adjoint) projection operators and the conditional probability is given by the von NeumannLüders formula [6]

$$
P(B \mid A)=\frac{\operatorname{tr}(B A W A)}{\operatorname{tr}(A W)}
$$

where $W$ is a density operator representing a quantum state. Since $P(A)=\operatorname{tr}(A W)$, we have

$$
P(A, B)=P(A) P(B \mid A)=\operatorname{tr}(B A W A)=\operatorname{tr}(A B A W) .
$$

In general, $P(A, B) \neq P(B, A)$ so $A$ and $B$ interfere. Stated differently, we can represent the sequential measurements $(A, B)$ and $(B, A)$ by the operators $A B A$ and $B A B$, respectively, and $(A, B) \neq(B, A)$ in general.

We say that two events $A$ and $B$ are sequentially independent if $(A, B)=$ $(B, A)$. Of course, $(A, B)=(B, A)$ if and only if $P(A, B)=P(B, A)$ for every probability measure $P$ or for every state $W$ in the quantum case. We have seen that classical events are always sequentially independent and would like to know when this happens for sharp quantum events. This is easily accomplished by letting $\psi$ be a vector satisfying $A \psi=\psi$. We then have

$$
\|B \psi\|^{2}=\langle B \psi, \psi\rangle=\langle A B A \psi, \psi\rangle=\langle B A B \psi, \psi\rangle=\langle A B \psi, B \psi\rangle .
$$

If $B \psi \neq 0$, then

$$
\left\langle A \frac{B \psi}{\|B \psi\|}, \frac{B \psi}{\|B \psi\|}\right\rangle=1 .
$$

It follows that $A B \psi=B \psi$. Hence, $B A=A B A$ and taking adjoints gives $A B=$ $A B A=B A$. Thus, $(A, B)=(B, A)$ if and only if $A$ and $B$ are compatible (or simultaneously measurable) and this reduces to the classical theory when only $A$ and $B$ are considered.

Notice that sequential independence is unrelated to stochastic independence which says that $P(B \mid A)=P(B)$. In the classical case, this means that $P(A \cap B)=$ $P(A) P(B)$. In the quantum case, we would have

$$
\operatorname{tr}(A B A W)=\operatorname{tr}(A W) \operatorname{tr}(B W) .
$$

This last equation practically never holds and is rarely considered. Notice also that stochastic independence is a symmetric relation in classical probability while it is not in quantum probability.

The main goal of this paper is the study of sequentially independent quantum effects. An effect corresponds to a quantum event that may be unsharp and is represented by a linear operator $A$ on a Hilbert space $H$ that satisfies $0 \leq A \leq I$. That is,

$$
0 \leq\langle A \psi, \psi\rangle \leq\langle\psi, \psi\rangle
$$


for all $\psi \in H$. Effects play an important role in quantum measurement theory and have been intensely studied [1, 2, 3]. We can already see one reason why effects are important from our previous discussion. Even if $A$ and $B$ are projections representing sharp events, the sequential measurement $B A B$ is not a projection unless $A$ and $B$ are compatible. However, $0 \leq B A B \leq I$ so $B A B$ represents an unsharp event in general.

\section{Sequential independent efFects}

Let $H$ be a complex Hilbert space and let $B(H)$ be the set of bounded linear operators on $H$. An operator $A \in B(H)$ is positive if $A \geq 0$; that is, $\langle A \psi, \psi\rangle \geq 0$ for all $\psi \in H$. It is easy to show that a positive operator is self-adjoint. Recall that $B \in B(H)$ is normal if $B B^{*}=B^{*} B$. In the sequel we shall need the following theorem due to Fuglede, Putnam and Rosenblum [5].

Theorem 1. Let $M, N, A \in B(H)$ with $M$ and $N$ normal. If $M A=A N$, then $M^{*} A=A N^{*}$.

Let $E, F \in B(H)$ be effects. Then $E^{1 / 2} F E^{1 / 2}$ is an effect because

$$
\begin{aligned}
0 \leq\left\langle E^{1 / 2} F E^{1 / 2} \psi, \psi\right\rangle & =\left\langle F E^{1 / 2} \psi, E^{1 / 2} \psi\right\rangle \leq\left\langle E^{1 / 2} \psi, E^{1 / 2} \psi\right\rangle \\
& =\langle E \psi, \psi\rangle \leq\langle\psi, \psi\rangle
\end{aligned}
$$

for every $\psi \in H$. The von Neumann-Lüders formula is generalized to effects [4] by defining

$$
P(F \mid E)=\frac{\operatorname{tr}\left(F E^{1 / 2} W E^{1 / 2}\right)}{\operatorname{tr}(E W)}=\frac{\operatorname{tr}\left(E^{1 / 2} F E^{1 / 2} W\right)}{\operatorname{tr}(E W)} .
$$

We call $E^{1 / 2} F E^{1 / 2}$ the sequential effect for $(E, F)$ and say that $E$ and $F$ are sequentially independent if

$$
E^{1 / 2} F E^{1 / 2}=F^{1 / 2} E F^{1 / 2} .
$$

We now prove a general result from which a characterization of sequential independence follows.

Theorem 2. For $A, B \in B(H)$ the following statements are equivalent.

(a) $A B^{*} B A^{*}=B A^{*} A B^{*}$ and $A^{*} B B^{*} A=B^{*} A A^{*} B$.

(b) $B A^{*} A=A A^{*} B$ and $A B^{*} B=B B^{*} A$.

Proof. To show that (b) implies (a), suppose that (b) holds. We then have

$$
A B^{*} B A^{*}=B B^{*} A A^{*}=B\left(A A^{*} B\right)^{*}=B\left(B A^{*} A\right)^{*}=B A^{*} A B^{*}
$$

and

$$
A^{*} B B^{*} A=A^{*} A B^{*} B=\left(B A^{*} A\right)^{*} B=\left(A A^{*} B\right)^{*} B=B^{*} A A^{*} B .
$$

To show that (a) implies (b), suppose that (a) holds and let $M=A B^{*}, N=B^{*} A$. It follows from (a) that $M$ and $N$ are normal and we have

$$
M A=A B^{*} A=A N .
$$

Applying Theorem 1 gives

$$
B A^{*} A=M^{*} A=A N^{*}=A A^{*} B .
$$


Now let $M=B A^{*}, N=A^{*} B$. It follows from (a) that $M$ and $N$ are normal and we have

$$
M B=B A^{*} B=B N
$$

Again, applying Theorem 1 gives

$$
A B^{*} B=M^{*} B=B N^{*}=B B^{*} A .
$$

Corollary 3. Two effects, $E$ and $F$, are sequentially independent if and only if $E F=F E$.

Proof. It is well known that if $E F=F E$, then $E^{1 / 2} F^{1 / 2}=F^{1 / 2} E^{1 / 2}[5$ and it follows that $E$ and $F$ are sequentially independent. Conversely, suppose that $E$ and $F$ are sequentially independent and let $A=E^{1 / 2}, B=F^{1 / 2}$. We then have that $A B^{2} A=B A^{2} B$ so by Theorem 2, $B A^{2}=A^{2} B$. It follows that $B^{2} A^{2}=A^{2} B^{2}$ and hence $E F=F E$.

We next consider three effects $E_{1}, E_{2}, E_{3}$. The natural definition for the probability of the sequential measurement $\left(E_{1}, E_{2}, E_{3}\right)$ becomes

$$
\begin{aligned}
P\left(E_{1}, E_{2}, E_{3}\right) & =P\left(E_{1}\right) P\left(E_{2} \mid E_{1}\right) P\left(E_{3} \mid\left(E_{1}, E_{2}\right)\right) \\
& =\operatorname{tr}\left(E_{1}^{1 / 2} E_{2}^{1 / 2} E_{3} E_{2}^{1 / 2} E_{1}^{1 / 2} W\right) .
\end{aligned}
$$

We then call $E_{1}^{1 / 2} E_{2}^{1 / 2} E_{3} E_{2}^{1 / 2} E_{1}^{1 / 2}$ the sequential effect for $\left(E_{1}, E_{2}, E_{3}\right)$. It is thus reasonable to say that $E_{1}, E_{2}, E_{3}$ are sequentially independent if

$$
E_{i}^{1 / 2} E_{j}^{1 / 2} E_{k} E_{j}^{1 / 2} E_{i}^{1 / 2}=E_{r}^{1 / 2} E_{s}^{1 / 2} E_{t} E_{s}^{1 / 2} E_{r}^{1 / 2}
$$

for every $i \neq j \neq k, r \neq s \neq t$. Moreover, we call $E_{1}, E_{2}, E_{3}$ permutable if

$$
E_{i} E_{j} E_{k}=E_{r} E_{s} E_{t}
$$

for every $i \neq j \neq k, r \neq s \neq t$.

Lemma 4. Effects $E, F, G$ are permutable if and only if $E^{1 / 2}, F^{1 / 2}, G^{1 / 2}$ are permutable.

Proof. Suppose that $E, F, G$ are permutable. In particular we have

$$
E F G=F E G=G E F=G F E .
$$

It follows that if $\psi$ is in the range $R(G)$ of $G$, then

$$
E F \psi=F E \psi \in R(G) .
$$

Since the closure $\bar{R}\left(G^{1 / 2}\right)=\bar{R}(G)$ we have $E F=F E$ on $\bar{R}\left(G^{1 / 2}\right)$ and hence $E^{1 / 2} F^{1 / 2}=F^{1 / 2} E^{1 / 2}$ on $\bar{R}\left(G^{1 / 2}\right)$. Therefore,

$$
E^{1 / 2} F^{1 / 2} G^{1 / 2}=F^{1 / 2} E^{1 / 2} G^{1 / 2}
$$

and taking adjoints gives

$$
G^{1 / 2} F^{1 / 2} E^{1 / 2}=G^{1 / 2} E^{1 / 2} F^{1 / 2} .
$$


The last two equations hold for all permutations of $E^{1 / 2}, F^{1 / 2}, G^{1 / 2}$. Hence,

$$
\begin{aligned}
F^{1 / 2} G^{1 / 2} E^{1 / 2} & =F^{1 / 2} E^{1 / 2} G^{1 / 2}=E^{1 / 2} F^{1 / 2} G^{1 / 2}=E^{1 / 2} G^{1 / 2} F^{1 / 2} \\
& =G^{1 / 2} E^{1 / 2} F^{1 / 2}=G^{1 / 2} F^{1 / 2} E^{1 / 2}
\end{aligned}
$$

so $A^{1 / 2}, B^{1 / 2}, C^{1 / 2}$ are permutable. The converse is similar.

We now characterize sequential independence for three effects.

Theorem 5. Effects $E, F, G$ are sequentially independent if and only if $E, F, G$ are permutable.

Proof. Suppose that $E, F, G$ are permutable. By Lemma $4 E^{1 / 2}, F^{1 / 2}, G^{1 / 2}$ are permutable. We then have

$$
E^{1 / 2} F^{1 / 2} G F^{1 / 2} E^{1 / 2}=E^{1 / 2} F^{1 / 2} G^{1 / 2} G^{1 / 2} F^{1 / 2} E^{1 / 2}=E^{1 / 2} G^{1 / 2} F G^{1 / 2} E^{1 / 2} .
$$

Since the other permutations are similar, we conclude that $E, F, G$ are sequentially independent.

Conversely, suppose that $E, F, G$ are sequentially independent. Letting $A=$ $E^{1 / 2}, B=F^{1 / 2}, C=G^{1 / 2}$ we have

$$
A B C^{2} B A=C B A^{2} B C .
$$

Letting $N=A B C$ and $M=B C A$ we have that $N$ and $M$ are normal and $N A=$ $A M$. Applying Theorem 1 gives $N^{*} A=A M^{*}$ and hence $C B A^{2}=A^{2} C B$. Since $A \geq 0$, it follows that $C B A=A C B$ [5]. We conclude that all cyclic permutations coincide. We now show that transpositions coincide; that is, $C A B=C B A$. To accomplish this, we have

$$
A^{2} B C^{2} B=A B C A C B=A B C^{2} B A=B C A^{2} C B=B C^{2} B A^{2} .
$$

Again, since $A \geq 0$ we have that $A B C^{2} B=B C^{2} B A$. Hence, $C^{2} A B^{2}=B^{2} A C^{2}$. Since $B(B A C)=(B A C) B, B$ and $B A C$ are commuting normal operators. It follows that $B^{2} A C$ is normal and similarly $C A B^{2}$ is normal. Since $\left(B^{2} A C\right) C=$ $C\left(C A B^{2}\right)$, by Theorem 1 we have that $C A B^{2} C=C B^{2} A C$. Hence, $B^{2} C A C=$ $C A C B^{2}$. Since $B \geq 0$, we conclude that $B C A C=C A C B$. Therefore,

$$
C^{2} A B=C A B C=B C A C=C A C B=C^{2} B A
$$

Letting $M=A B-B A$ we have that $C^{2} M=0$ and thus $M^{*} C^{2} M=0$. Hence, for every $\psi \in H$ we have

$$
\|C M \psi\|^{2}=\langle C M \psi, C M \psi\rangle=\left\langle M^{*} C^{2} M \psi, \psi\right\rangle=0
$$

We conclude that $C M=0$ so that $C A B=C B A$. By symmetry, all transpositions coincide so $A, B, C$ are permutable. Applying Lemma 4, we conclude that $E, F, G$ are permutable.

Comparing Corollary 3 and Theorem [5] we see that $E, F$ are sequentially independent if and only if $E$ and $F$ are compatible while $E, F, G$ are sequentially independent if and only if $E, F, G$ are only permutable. It is interesting to note that three effects that are permutable need not be mutually compatible even if 
they are sharp (that is, projections). For example, let $E, F, G$ be the following projections on $\mathbb{C}^{3}$ :

$$
E=\left[\begin{array}{lll}
1 & 0 & 0 \\
0 & 0 & 0 \\
0 & 0 & 0
\end{array}\right], \quad F=\left[\begin{array}{lll}
0 & 0 & 0 \\
0 & 1 & 0 \\
0 & 0 & 0
\end{array}\right], \quad G=\frac{1}{2}\left[\begin{array}{lll}
0 & 0 & 0 \\
0 & 1 & 1 \\
0 & 1 & 1
\end{array}\right] .
$$

Thus, three effects that are sequentially independent need not be compatible in pairs. However, by employing the methods in Lemma 4 we do have the following result.

Corollary 6. Effects $E_{1}, E_{2}, E_{3}$ are sequentially independent if and only if $E_{i} E_{j}=$ $E_{j} E_{i}$ on $R\left(E_{k}\right)$ for all $i \neq j \neq k$.

This work can be continued for four or more effects and a result similar to Theorem 5 holds.

\section{REFERENCES}

[1] P. Busch, P. J. Lahti and P. Mittlestaedt, The Quantum Theory of Measurements, SpringerVerlag, Berlin, 1991. MR 93m:81014

[2] P. Busch, M. Grabowski and P. J. Lahti, Operational Quantum Physics, Springer-Verlag, Berlin, 1995. MR 96j:81022

[3] E. B. Davies, Quantum Theory of Open Systems, Academic Press, London, 1976. MR 58:8853

[4] S. Gudder, A histories approach to quantum mechanics, J. Math. Phys. 39 (1998), 5772-5788. MR 99h:81011

[5] W. Rudin, Functional Analysis, McGraw-Hill, New York, 1991. MR 92k:46001

[6] J. von Neumann, Mathematical Foundations of Quantum Mechanics, Princeton University Press, Princeton, New Jersey, 1955. MR 16:654a

Department of Mathematics, University of Denver, Denver, Colorado 80208

E-mail address: sgudder@cs.du.edu

Department of Mathematics, Kansas State University, Manhattan, Kansas 66506

E-mail address: nagy@math.ksu.edu 